\title{
O Problema da Consciência: por Onde Começar o Estudo?
}

\author{
The Problem of Consciousness: Where to Start \\ Studying?
}

\author{
Marselle Soares S. Klem de Mattos \\ Gabriel Pereira da Silva ${ }^{I I}$ \\ Renato Faria da Gama ${ }^{I I I}$
}

\section{PALAVRAS-CHAVE}

- Consciência.

- Cérebro.

- Mente.

- Neurociência.
I Universidade Estadual do Norte Fluminense Darcy Ribeiro, Campos dos Goytacazes, Rio de Janeiro, Brasil.

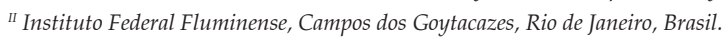

III Santa Casa de Misericórdia de Campos dos Goytacazes, Campos dos Goytacazes, Rio de Janeiro, Brasil. 


\section{KEY-WORDS}

- Consciousness

- Brain.

- Mind.

- Neuroscience.

Recebido em: 1/8/2018

Aceito em: 26/9/2018

\begin{abstract}
The study of the nervous system and its functions is fascinating and complex. One of the most intriguing in this field is how neurobiology produces conscious states; the notorious problem of consciousness (the brain and mind). For this study, a knowledge of both neuroanatomy and physiology is required, as well as of linguistic and philosophical issues. For this reason, this is a topic that is feared or avoided by medical students. The student wishing to begin this chapter of neuroscience faces a question: where to start? There is a vast amount of information on the subject, and the starting point, in general, can be biology, psychology or philosophy. This essay suggests one of several possible means of guiding those seeking preliminary information on the subject. Some students have difficulty with the subject because they lack the fundamental concepts needed, or they do not know the theories defended, but are focused on subject from a purely biological perspective. This work begins by presenting three conceptual possibilities for the word 'conscience', and some language limitations related to it. Next, it gives a succinct overview of neurobiology, with emphasis on neuronal electrochemical activity. The theories of consciousness are outlined, giving the points of view of two authors: David Chalmers and Daniel Dennett. This choice of authors demonstrates that such theories have pendular movement: starting with Descartes and his dualist theory at one extreme, passing through other intermediate theories, and reaching the reductionist materialists at the opposite extreme. The methodology consists of a literature search of scientific articles on the subject. Finally, although the majority argue that consciousness is a biochemical phenomenon, because in order to have consciousness there must be a brain, there are also those who argue that consciousness is not an attribute of matter. Discussions like these should be encouraged, and based on theoretical studies.
\end{abstract}

\section{CONSIDERAÇÕES INICIAIS}

As neurociências têm atraído crescente quantidade de estudantes que desejam atuar como pesquisadores nesta área ou obter mais informações para o embasamento científico de seus trabalhos. Devido ao seu caráter interdisciplinar, as neurociências englobam abordagens de variados ramos do conhecimento. De acordo com o neurocientista Lent ${ }^{1}$, no prefácio do livro Cem Bilhões de Neurônios, a neurociência nasceu da necessidade de integrar as diversas áreas das ciências biológicas para a compreensão do funcionamento do sistema nervoso (por exemplo: neuroanatomia, neurofisiologia e neurologia). Inicialmente, tinham como ator principal o cérebro.

Com o passar dos anos, a mente também se tornou alvo das investigações científicas, assim como a consciência, as emoções e o comportamento. Deste modo, a interação cérebro e mente passou a ser o cerne desses estudos, motivando o desenvolvimento de novas tecnologias para avaliação da atividade neuronal e intensificando as discussões. Contudo, não são contemporâneas as discussões relacionadas à atividade cerebral e à associação do cérebro com a mente. Desde a Antiguidade, este tema provoca indagações e controvérsias, primeiramente por parte dos filósofos, e continua até a atualidade com a neurociência moderna.

Um dos assuntos instigantes das neurociências é a consciência. $\mathrm{O}$ estudo desta questão tem vasta abrangência, podendo se iniciar com o conceito da palavra consciência, passar pelos fundamentos da neurobiologia e chegar às questões das teorias de como o cérebro gera os fenômenos mentais. Além desses aspectos, há discussões em torno da filosofia da ciência, que se encarrega de analisar a metodologia, a interpretação das descobertas científicas, possíveis vieses e outras questões deveras importantes, mas que extrapolam o foco deste trabalho.

Portanto, como problematizado no título deste artigo, aqueles que desejam iniciar o estudo deste tema podem não saber por onde começar, frente à grande quantidade de informações. Parte-se da hipótese de que, algumas vezes, iniciam estudando discussões sem terem maturidade acadêmica no tema, o que dificulta a compreensão do assunto. Logo, este ensaio tem por objetivo oferecer um ponto de partida àqueles que almejam iniciar o estudo da consciência. Não se destina aos especialistas na área, visto que serão abordados conceitos básicos e apresentadas algumas questões elementares. 
Destaca-se, por último, que este trabalho se limita apenas a uma sugestão, pois são diversos os caminhos de estudo e autores que abordam o tema da consciência. Entre esses vários autores, destacam-se pesquisadores de diferentes formações acadêmicas, como biólogos, físicos, filósofos, médicos e psicólogos, caracterizando a interdisciplinaridade do tema.

\section{ALGUNS CONCEITOS SOBRE CONSCIÊNCIA}

Para iniciar os estudos sobre consciência, antes das discussões neurocientíficas, é importante entender o significado dessa palavra, que, de acordo com o idioma, pode gerar confusão em relação ao que se deseja expressar. No livro Consciousness - A User's Guide ${ }^{2}$, Zeman propõe uma análise semântica da palavra no idioma inglês. A palavra mãe das demais seria conscience, originada pela junção de duas expressões em latim: cum (com) e scio (eu sei / eu conheço). Assim, derivaram o adjetivo conscius (aware), o verbo conscio e o substantivo consciouness (awareness). Por fim, o autor conclui que o problema da consciência surge de uma limitação do uso da linguagem: "If there are any 'problem of consciousness' they arise from our inconsistent and unsophisticated use of language" ${ }^{\prime 2}$. (p. 14).

O significado de "consciência" (consciouness ou awareness) no idioma português gera mais confusões em comparação ao inglês. Provavelmente, por haver apenas uma palavra para designar diferentes significados (um type para pelo menos três tokens): consciência neurológica, consciência psicológica e consciência moral ou de responsabilidade. Quando dizemos que alguém está consciente, em princípio, queremos dizer que a pessoa está acordada, vígil (awake). Estamos nos referindo ao primeiro token da palavra consciência: ao estado de consciência, uma análise neurológica (orgânica). A utilização das expressões type e token faz parte de estudos metafísicos ${ }^{3}$. Neste exemplo, o type é a palavra consciência, e os tokens são seus possíveis significados.

Em neurologia, frente a um trauma cranioencefálico, por exemplo, o nível de consciência pode ser avaliado por um parâmetro objetivo, a escala de coma de Glasgow (ECG). Essa escala avalia o nível de consciência neurológica por parâmetros: abertura dos olhos, resposta motora e resposta verbal. Cada resposta ao exame físico resulta numa pontuação. A pontuação mais alta é 15, e 8 é estado de coma ${ }^{4}$.

Contudo, logo surge uma encruzilhada semântica: o indivíduo pode estar acordado e não estar consciente. A questão não é que a pessoa se apresente em dois estados de consciência (acordada e desacordada ou consciente e inconsciente) ao mesmo tempo. Neste caso, o indivíduo pode estar acordado, mas não ter consciência de quem é, por exemplo. Isto ocorre devido a uma limitação linguística para a correta expressão do fato, pois se utiliza uma única palavra para mais de um significado.
Estar consciente nos dois sentidos abordados acima significa o estado de vigília que permite à pessoa perceber e processar os estímulos de forma que tenha condições de orientar-se quanto a sua condição pessoal. É o estado de consciência que permite outra atribuição mental, a orientação, que é classificada em autopsíquica (de si próprio) e alopsíquica (relacionada à orientação do sujeito no tempo e no espaço) $)^{5}$.

Assim, diante desses dois primeiros significados da palavra consciência, pode-se perceber que, enquanto a neurologia busca utilizar um parâmetro mais objetivo, para a psicologia a consciência pode ser entendida como "[...] a soma total das experiências conscientes do indivíduo em um determinado momento. É a dimensão subjetiva da atividade psíquica do sujeito que se volta para a realidade ${ }^{\prime \prime 6}$ (p. 104).

$\mathrm{O}$ terceiro sentido atribuído à consciência é evidenciado quando o indivíduo tem conhecimento do significado de determinadas atitudes e deliberadamente opta por elas. Essa escolha consciente seria resultado da capacidade cognitiva e formação cultural. Exemplificando, quando alguém faz algo que é reprovado pelo padrão social, lhe é perguntado: você tem consciência do que fez? O que se pretende indagar é se a pessoa sabe das implicações de seus atos.

Visivelmente, os homens são capazes de melhor responder aos eventos que ocorrem no ambiente externo quando estão conscientes. Essa capacidade, chamada de consciência, é indispensável à vida. Identificar objetos, interpretar se algo representa perigo, se determinado bicho é um predador em potencial ou não, interagir com as fêmeas, selecionar alimentos são necessidades requeridas para a sobrevivência da espécie.

Desta maneira, entende-se que a consciência pertence ao mesmo domínio da mente numa relação de interdependência, ou seja, a consciência é caracterizada pela presença da mente, visto que os sujeitos estão conscientes de diversos eventos que passam pela mente.

Em suma, é necessário estar desperto e com condições de perceber e entender os estímulos, para então adotar determinados comportamentos e ações, e, assim, poder compreender as consequências de seus atos. Esta é a consciência da responsabilidade ou consciência moral. "Thus the third sense of consciousness bridges perception and action. The events we perceive and the ones we bring about"2 (p. 21).

As questões relacionadas à escolha consciente de determinadas atitudes e ações fazem parte de outra discussão, sobre o livre-arbítrio. E há autores ${ }^{7}$ que afirmam que não temos esta liberdade de escolha, pois já nascemos com uma programação genética para a atividade cerebral, isto é, o cérebro trabalha de forma incógnita. 


\section{A BIOLOGIA CEREBRAL}

A biologia do sistema nervoso, central e periférico é complexa e dinâmica. Mas, entre as disciplinas que compõem as neurociências, os enfoques que possuem informações e resultados de mais fácil compreensão e menos controversos provavelmente são a anatomia e a fisiologia do sistema nervoso, assuntos desta parte.

Pode-se começar a estudar a neurobiologia partindo das estruturas anatômicas (macro), passando pelas estruturas celulares (micro) e chegando até às estruturas moleculares, ou vice-versa. Neste artigo, a preferência é iniciar por uma visão geral do sistema nervoso até chegar aos neurônios.

Resumidamente, o sistema nervoso se divide em central e periférico. $\mathrm{O}$ central é composto pela medula e pelo encéfalo. O encéfalo é dividido didaticamente em cérebro (telencéfalo e diencéfalo), tronco encefálico (mesencéfalo, ponte e bulbo) e cerebelo. No cérebro podem-se identificar os lobos frontal, parietal, temporal e occipital ${ }^{8}$.

O córtex cerebral (substância cinzenta) é onde estão situados os corpos dos neurônios. No início do século XX, o neurocientista Korbinian Brodman iniciou o trabalho de mapeamento do córtex de acordo com características funcionais e motoras distintas. "Por exemplo, a linguagem, atributo dos mais fundamentais para os seres humanos, ocupa cerca de $20 \%$ das áreas corticais do hemisfério esquerdo ${ }^{\prime \prime 9}$ (p. 147).

A consciência, o pensamento, a emoção e o comportamento, assim como toda a atividade cognitiva são atributos da atividade cerebral mediados por células nervosas, os neurônios (como será visto adiante, esta é uma teoria fisicalista). Estas células têm as funções de recepção, integração e transmissão de informações a outros neurônios, por meio de sinalização elétrica e química (sinapses), mediada por neurotransmissores ${ }^{4}$.

As informações oriundas do meio externo ou interno do corpo chegam e saem do cérebro pelos neurônios, que se organizam em sistemas sensoriais e motores. Os sistemas sensoriais captam estímulos e os transformam em impulsos neuronais. Já os sistemas motores têm como principal função os movimentos musculares corporais: "No cérebro, as informações sensoriais, representando o mundo externo, são integradas aos impulsos internos, às memórias e aos estímulos emocionais em unidades de associação, que, por sua vez, dirigem as ações das unidades motoras" ${ }^{\prime 5}$ (p. 4).

Desta forma, percebe-se que doenças orgânicas, traumas e tumores, por exemplo, podem alterar a anatomia e a bioquímica cerebral, resultando em alterações que acarretam uma disfunção na atividade dos neurônios. Este fato pode resultar em sintomas físicos, psíquicos e alterações comportamentais. Em contrapartida, também existem pessoas que não possuem uma condição patológica, mas exibem comportamentos desviantes do padrão para determinada realidade.

Esses desvios comportamentais em pessoas sem doença diagnosticada que os explique também são temas de pesquisas em neurociências. Mais especificamente da neurociência cognitiva, que utiliza como uma das ferramentas para esses estudos técnicas de neuroimagem funcional. Essas neuroimagens são obtidas frente a uma atividade solicitada, como, por exemplo, ouvir uma música, ver uma fotografia ou pensar em algo proposto pelos pesquisadores.

Outro fato instigante, ainda na seara dos fatores que influenciam a atividade cerebral, gerando respostas orgânicas ou comportamentais, é o efeito placebo. Pesquisas ${ }^{10}$ mostram que em algumas pessoas que ingerem uma pílula de farinha acreditando ser um fármaco para tratar determinado sintoma, esse pensamento acarreta a produção endógena de neurotransmissores que acabam agindo positivamente nesse sintoma. Nesse caso, não foi o efeito biológico que interferiu na atividade cerebral e mental, mas, sim, o contrário, o pensamento interferiu na matéria orgânica.

Por fim, pela neurobiologia é possível demonstrar e compreender como o cérebro se conecta com o mundo externo e gera respostas frente aos estímulos. Entretanto, a biologia ainda não conseguiu explicar como a atividade cerebral gera a consciência. O que se tem até agora são teorias. Duas dessas teorias são apresentadas a seguir.

\section{PENSADORES DO PROBLEMA DA CONSCIÊNCIA}

De acordo com Frith e Rees ${ }^{11}$, o primeiro a estudar a questão cérebro e mente de forma sistematizada foi René Descartes no século XVI. Ele postulou a distinção feita entre o cérebro (res extensa) e a mente (res cogitans), batizada de dualismo cartesiano. Com o passar do tempo, filósofos e cientistas contestaram o dualismo cartesiano. Começaram a suspeitar que de alguma forma a mente emerge de propriedades físicas do cérebro. Mas o problema é como? Para tentar responder a essa pergunta, várias teorias foram propostas e podem ser agrupadas em dois grandes grupos: os dualistas e os monistas.

Para os dualistas, a mente não é derivada de atividade neurofisiológica, enquanto para os monistas a mente é um atributo da atividade cerebral. Entre os monistas também existe subdivisão, como os materialistas/fisicalistas, emergentistas e outros. A diferença principal seria que para os primeiros a mente é o cérebro (materialistas reducionistas), e para os emergentistas a mente emerge do funcionamento do cérebro ${ }^{12}$.

Os dualistas consideram a teoria cartesiana, em que a consciência é um fenômeno não físico. Levando em conta os avanços dos estudos neurocientíficos, à primeira vista, alguns 
podem julgar que esse tipo de pensamento ficou restrito ao passado. No entanto, cientistas cognitivos atuais defendem que o estado mental não advém de um estado físico cerebral. Nesse ínterim, o filósofo australiano David Chalmers defende que a consciência é composta por duas propriedades distintas, uma fenomenal e outra psicológica: "The division of mental properties into fenomenal and psychological properties has the effect of dividing the mind-body problem into two: an easy part and a hard $\operatorname{part}^{\prime \prime 13}$ (p. 21).

Para esse autor, o problema fácil está relacionado às propriedades psicológicas, que ele identifica como as características estruturais e funcionais. O problema difícil refere-se à propriedade fenomenal da consciência, ou às qualidades subjetivas da consciência resultantes da experiência individual. No contexto do problema difícil, Chalmers ${ }^{13}$ refere-se às propriedades qualitativas e subjetivas intrínsecas da consciência, também denominadas qualia. Para ele, qualia é uma propriedade não física, comprovando, assim, o retorno ao dualismo cartesiano.

No intuito de demonstrar que o materialismo fisicalista não é suficiente para explicar a consciência, Chalmers propõe a existência de um ser idêntico ao ser humano no sentido fisiológico, porém sem consciência, um zumbi. O ser faria tudo que um homem comum faz, mas não teria pensamentos, sentimentos, intencionalidade e nem consciência de si.

Esse argumento foi de pronto rebatido pelos materialistas. Entre eles, o mais conhecido é o filósofo americano Daniel Dennett, que propõe uma teoria fisicalista para a consciência. De acordo com Dennett, o dualismo foi suplantado pela inteligência artificial e pela evolução das neurociências. Em outras palavras, o estudo da consciência é o estudo do funcionamento cerebral.

Conforme Tavares ${ }^{14}$, para Dennett o que existe de fato são pensamentos e decisões tomadas a partir da atividade cerebral, logo os qualia não existem. Mas esta afirmação de que não existe qualia é um pouco arrojada, visto que, embora a tendência dos neurocientistas atuais seja voltada ao materialismo, a neurociência ainda não conseguiu confirmar ou negar seguramente a afirmação de Dennett.

Entre os extremos, desde a existência de qualia não física à negação da existência desta, é preferível uma postura mais cautelosa, em que se pode entender qualia como atributos da atividade cerebral: "Por outro lado, se qualia são propriedades vivenciadas fisicamente (por sujeitos que são, em última instância, agregados físicos), por conseguinte, os qualia devem ser propriedades físico-químicas produzidas sob certas condições pelo cérebro e vivenciadas subjetivamente por um sujeito"15 (p. 102).

As discussões não param por aqui. Mas, devido à necessidade de sintetizar o assunto, optou-se por abordar apenas al- gumas vertentes do tema proposto. A intenção é de que essas informações preliminares possam auxiliar no início dos estudos sobre o problema da consciência e instigar a continuidade.

\section{CONSIDERAÇÕES FINAIS}

Como se pode perceber, o problema da consciência é um tema extenso. O que se observa em geral são postulados que visam integrar as propriedades da consciência à biologia neural. Em alguns casos, praticamente um palpite do autor, pois não são apontadas metodologias para análise e replicação de experimentos que permitam evidenciar que a consciência emerge da atividade elétrica e química cerebral, como defendem os emergentistas. Embora, seja a possibilidade mais aceita.

Pesquisas em neurociência experimental são extremamente caras porque requerem uma equipe multidisciplinar, aparelhos cada vez mais modernos e atualizados, locais específicos com proteção para o ambiente externo, etc. E, por se tratar de uma ciência, os experimentos devem ser replicáveis, e cada sessão tem um custo muito elevado, pelos motivos citados.

A neurociência ainda passa pela fase da infância. Muitas teorias surgem, e há diferentes percepções e levantamentos com diferentes perspectivas, visto que o campo é interdisciplinar. Espera-se que, ao atingir a maturidade, possa nos fornecer respostas que permitam comprovação científica para o problema da consciência.

\section{REFERÊNCIAS}

1. Lent, R. Cem bilhões de neurônios: conceitos fundamentais de neurociência. São Paulo: Atheneu, 2010.

2. Zeman, A. Consciousness - A user's guide. London: Yale University Press Publication, 2004.

3. Balashov, Y; Rosenberg, A. Philosophy of Science. Contemporary Readings. London: Routledge, 2002.

4. Yudofsky, S; Hales, RE. Neuropsiquiatria e Neurociências na Prática Clínica. Porto Alegre: Artmed, 2006.

5. Sadock, BJ; Sadock, VA; Ruiz, P. Compêndio de Psiquiatria: ciência do comportamento e psiquiatria clínica. 11ª ed. Porto Alegre: Artmed, 2017.

6. Dalgalarrondo, P. Psicopatologia e semiologia dos transtornos mentais. Porto Alegre: Artmed, 2000.

7. Eagleman, D. Incognito. The secret lives of the brain. New York: Vintage Books, 2011.

8. Machado, A; Haertel, L, M. Neuroanatomia Funcional. $3^{\text {a }}$ ed. São Paulo: Atheneu, 2013. 363p.

9. Dalgalarrondo, P. Evolução do Cérebro. Sistema nervoso, psicologia e psicopatologia sob a perspectiva evolucionista. Porto Alegre: Artmed, 2011.

10. Goldacre, B. Ciência Picareta. Civilização brasileira, 2013. 
11. Frith, C.; Rees, G. A Brief History of the Scientific Approach to the Study of Consciousness. In: The Blackwell Companion to Consciousness. Malden: Blackwell Publishing, 2007. p. 7-22.

12. Victorino, CG. Uma Introdução à Neurofilosofia: O Problema Mente-Corpo. Revista da Biologia. Instituto de Biociências USP [online]. 2009. 3(1) [capturado 14 abr. 2018]; p.15-20. Disponível em http://www.ib.usp.br/revista/ node/28.

13. Chalmers, DJ. The Conscious Mind: In Search of a Fundamental Theory. Oxford: Oxford University Press, 1996.

14. Tavares, DR. A Busca de Solução Para o "Problema Difícil" da Consciência: Uma Revisão das Perspectivas de Chalmers, Dennett e Damásio. Revista Ciências \& Cognição [online].2018. 23 (1) [capturado 01 maio de 2018]; p.138157. Disponível em http://www.cienciasecognicao.org/ revista/index.php/cec/index

15. De Sousa, CEB. Modelos Neurais de Consciência: uma análise neurofilosófica. Trans/Form/Ação, 2015; 38 (2) 95-128.

\section{CONTRIBUIÇÃO DOS AUTORES}

Os três autores contribuíram de forma equivalente na pesquisa para a produção deste trabalho. A redação foi elaborada pela autora 1 .

\section{CONFLITO DE INTERESSES}

Não há conflito de interesse por parte dos autores. São estudantes e profissionais liberais.

\section{ENDEREÇO PARA CORRESPONDÊNCIA}

Rua Bariloche, 212. Sala 301. Cavaleiros. Macaé/RJ. CEP: $27.920-160$ 